\title{
Miocardiopatía ventricular derecha: un diagnóstico a tener en cuenta
}

\author{
A. DOMÍNGUEZ RODRÍGUEZ, A. BARRAGÁN ACEA, M.J. GARCÍA GONZÁLEZ, \\ A. LARA PADRÓN, D. DE ARMAS TRUJILLO
}

Servicio de Cardiología. Hospital Universitario de Canarias. La Laguna.

Santa Cruz de Tenerife

\author{
RIGHT VENTRICULAR CARDIOMYOPATHY: A DIAGNOSIS TO \\ BEAR IN MIND
}

\begin{abstract}
RESUMEN
La miocardiopatía arritmogénica ventricular derecha es una entidad de etiopatogenia desconocida, que patológicamente se caracteriza por atrofia miocárdica ventricular derecha y sustitución por tejido fibroadiposo. Presentamos un paciente de 65 años de edad diagnosticado de hepatopatía crónica, cuyo estudio llevó al diagnóstico de miocardiopatía arritmogénica ventricular derecha.
\end{abstract}

PALABRAS CLAVE: Miocardiopatía arritmogénica de ventrículo derecho. Insuficiencia cardíaca. Taquicardia ventricular.

\begin{abstract}
Arrhythmogenic right ventricular cardiomyopathy is a entity of unknown etiology, that is pathologically characterized by right ventricular myocardial atrophy and fibroadipous tissue replacement. We present the case of a 65-year-old male patient with the diagnosis of chronic liver disease, whose study lead a diagnosis of Arrhythmogenic right ventricular cardiomyopathy.
\end{abstract}

KEY WORDS: Arrhythmogenic right ventricular dysplasia/cardiomyo pathy. Heart failure. Ventricular tachycardia.

Domínguez Rodríguez A, Barragán Acea A, García González MJ, Lara Padrón A, de Armas Trujillo D. Miocardiopatia Ventricular Derecha: un diagnostico a tener en cuenta. An Med Interna (Madrid) 2001; 18: 323-325.

\section{INTRODUCCIÓN}

La miocardiopatía o displasia arritmogénica ventricular derecha es una enfermedad del músculo cardiaco que se caracteriza por una disminución de los elementos contráctiles de la pared del ventrículo derecho y su sustitución por tejido adiposo o fibroadiposo (1). Su incidencia y prevalencia en la población general es desconocida. Presentamos el caso de un paciente afecto de miocardiopatía ventricular derecha arritmogénica diagnosticado de hepatopatía crónica.

\section{CASO APORTADO}

Se trata de un paciente varón de 65 años de edad, diagnosticado de hepatopatía crónica de probable origen enólico, en tratamiento con furosemida y espironolactona. Acudió al Servicio de Urgencias por disnea intensa de reposo, intolerancia al decúbito y edemas en miembros inferiores. Entre sus antecedentes personales destacaba ser fumador de 1,2 paquetes de cigarrillos por día, etilismo crónico de 35 gramos de alcohol por día, obstrucción crónica al flujo aéreo, y hemorragia digestiva alta por lesiones agudas de la mucosa gástrica en febrero de 1997. Desde hacía aproximadamente 2 años presentaba disnea a esfuerzos moderados, distensión abdominal y edemas en miembros inferiores.
La exploración física al ingreso mostró: hipotensión arterial (80/50), acrocianosis, tolerancia parcial al decúbito, taquipnea, e ingurgitación venosa yugular "pasiva" ++++/++++ a $45^{\circ}$. La exploración cardiológica reveló latido de la punta desplazado ligeramente hacia abajo y en línea axilar anterior, y unos ruidos cardiacos rítmicos de baja intensidad, taquicárdicos, sin soplos, ni roce; en la auscultación pulmonar disminución del murmullo vesicular en tercio inferior de hemitoráx izquierdo, que se encontraba abolido en los dos tercios inferiores de hemitoráx derecho, con soplo pleural a ese nivel. En el abdomen existía hepatomegalia no pulsátil de $4-5 \mathrm{~cm}$. bajo reborde costal derecho, oleada ascítica y ruidos intestinales presentes. Presentaba edemas en ambas rodillas y tobillos $++/+++$. La analítica sanguínea se encontró con parámetros dentro de límites normales, excepto plaquetas: $115 \times 10^{9} / \mathrm{l}$, creatinina: $1,6 \mathrm{mg} / \mathrm{dl}$, albúmina: $3,3 \mathrm{gr} / \mathrm{dl}$, bilirrubina total: $1,4 \mathrm{mg} / \mathrm{dl}$ (directa: 0,8; indirecta: 0,6), y GGT $116 \mathrm{U} / \mathrm{l}$. El electrocardiograma mostró taquicardia de QRS ancho a $180 \mathrm{lpm}$, eje $+60^{\circ}$ y morfología de bloqueo completo de rama izquierda, que tras cardioversión con 200 julios, pasó a ritmo sinusal con un eje $+120^{\circ}$ y trastornos difusos de la repolarización ventricular. La Rx de tórax objetivó una silueta cardiaca aumentada de tamaño por crecimiento de cavidades derechas y derrame pleural bilateral con predominio en el lado derecho. Se le realizó un Eco 2D y M que mostró un ventrículo izquierdo ligeramente hipertrófico con cavidad pequeña, dilatación importante de cavidades derechas, y disfunción diastólica y sistólica del ventrículo derecho. Se realizó toracocentesis evacuadora y se esta-

Trabajo aceptado: 30 de Septiembre de 1999

Correspondencia: Alberto Domínguez Rodríguez. Servicio de Cardiología. Hospital Universitario de Canarias. Ofra s/n. La Cuesta. 38320 La Laguna. Santa Cruz de Tenerife. 


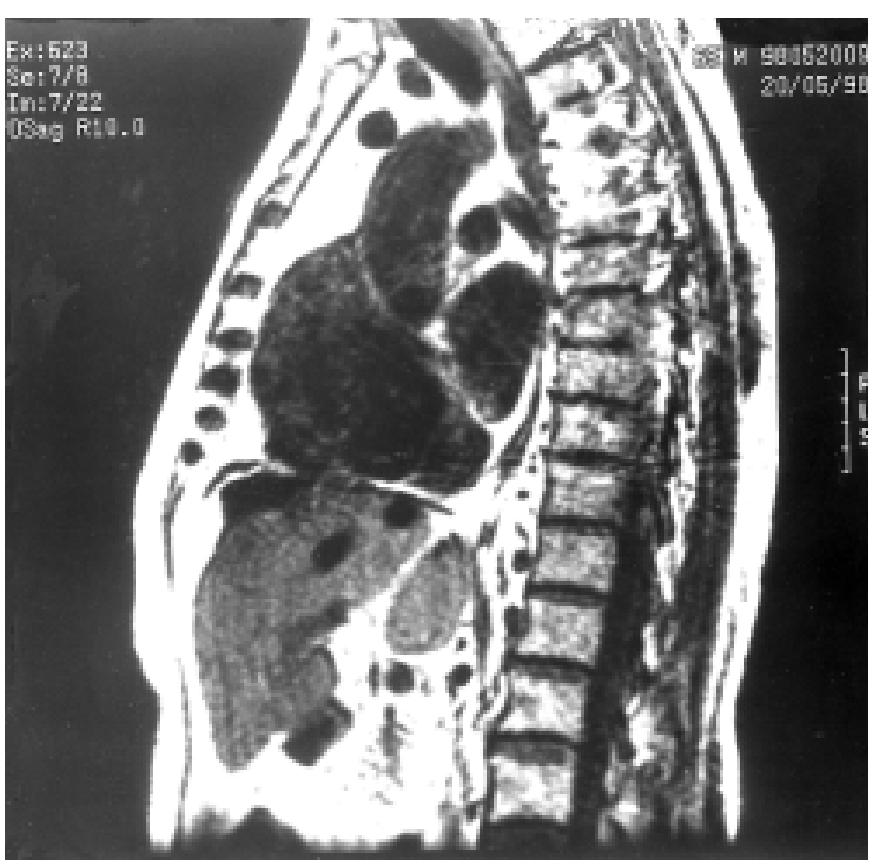

Fig. 1. Resonancia magnética nuclear. Dilatación de ventrículo y aurícula derecha, con áreas de infiltración grasas.

bilizó el cuadro clínico con tratamiento médico (diuréticos y aminas). Posteriormente se le realizó estudio hemodinámico y angiográfico que mostró: arterias coronarias normales, ventrículo izquierdo muy deformado con función sistólica conservada, y dilatación del ventrículo derecho con reducción de su contractilidad. Por último y con el fin de completar el estudio, se le practicó una resonancia magnética nuclear (Fig. 1) que evidenció una importante dilatación tanto del ventrículo como de la aurícula derechos, con significativo adelgazamiento de la pared del ventrículo derecho, sugestivo de áreas de infiltración grasa, hallazgos compatibles con la existencia de una miocardiopatía arritmogénica de ventrículo derecho (MAVD), para cuyo diagnóstico el paciente reúne suficiente criterios.

\section{DISCUSION}

Como es sabido, la insuficiencia cardiaca derecha crónica puede dar origen a una hepatopatía crónica, debido a la transmisión retrógrada de la presión venosa elevada a través de la vena cava inferior y de las venas hepáticas que produce congestión hepática, y que en los casos de larga evolución puede conducir a cirrosis hepática. En muchas ocasiones es un reto para el clínico filiar la etiología de ésta última cuando el paciente se presenta en un estado avanzado de la enfermedad, por no ser muy evidentes, o incluso estar enmascarados signos inequívocos de insuficiencia cardiaca derecha, como son la hipertensión venosa yugular, por el uso de terapia diurética habitualmente utilizada en estas situaciones. Aportamos el caso de un paciente varón diagnosticado de hepatopatía crónica por semiología ascítico-edematosa de probable origen etílico, que acudió a urgencias por cuadro de insuficiencia cardiaca derecha severo y taquicardia ventricular sostenida en el que más tarde se demostró que padecía una MAVD.

La MAVD es una enfermedad que se caracteriza por la sustitución progresiva de las fibras del ventrículo derecho por
TABLA I

CRITERIOS PARA EL DIAGNÓSTICO DE M AVD

A) Disfunción global o regional y cambios estructurales (detectados por ecocardiografía, angiografía, resonancia magnética o ventriculografía isotópica)

\section{M ayores}

Dilatación severa y reducción de la fracción de eyección del VD sin afectación (o sólo ligera) del VI

Aneurismas localizados del VD (áreas acinéticas o discinéticas con abultamiento diastólico)

Dilatación severa del VD

\section{Menores}

Ligera dilatación global del VD y/o reducción de la fracción de eyección con VI normal

Dilatación ligera y segmentaria del VD

Hipocinesia regional del VD

\section{B) Características tisulares}

\section{Mayores}

Sustitución del tejido miocárdico por tejido fibroadiposo en la biopsia endomiocárdica

\section{C) Anomalías de la repolarización}

\section{Menores}

$\mathrm{T}$ invertidas en las derivaciones precordiales derechas (V2 y V3) (individuos de más de 12 años y en ausencia de bloqueo completo de rama derecha)

\section{D) Anormalidades de la despolarización/ conducción}

\section{Mayores:}

O ndas epsilón o prolongación localizada (> $110 \mathrm{~ms}$ ) del

Q RS en las derivaciones precordiales derechas (V1-V3)

\section{Menores}

Potenciales tardíos (en el electrocardiograma de señal promediada)

\section{E) Arritmias}

\section{Menores}

Taquicardia ventricular con patrón de bloqueo de rama izquierda (sostenida o no sostenida) (en el ECG, Holtter, ergometría)

Extrasistolia ventricular frecuente ( $>1000 / 24 \mathrm{~h})$ (Holter)

\section{F) Historia familiar}

\section{Mayores}

Enfermedad familiar confirmada en la autopsia o en cirugía

\section{Menores}

Historia familiar de muerte súbita prematura (<35 años) con sospecha de displasia arritmogénica de VD

Historia familiar (diagnóstico clínico basado en los criterios expuestos)

VD: Ventrículo Derecho. VI: Ventrículo izquierdo. 
tejido adiposo. La MAVD es progresiva y puede presentar varios patrones clinicopatológicos: 1) una forma "silente" donde existen anormalidades miopáticas localizadas en el ventrículo derecho en víctimas asintomáticas de muerte súbita, 2) otra forma caracterizada por cambios estructurales en el ventrículo derecho que se afecta de forma segmentaria o global y cursa con arritmias ventriculares sintomáticas y; 3) un estado final que afecta a ambos ventrículos, y se asemeja a una miocardiopatía dilatada, que conduce a insuficiencia cardiaca progresiva y puede requerir trasplante cardiaco (2). Su presentación clínica habitual consiste en la presencia de crisis de taquicardia ventricular monomórfica, donde se observa electrocardiográficamente un patrón de bloqueo de rama izquierda. Igualmente la enfermedad, en algunos casos, se puede manifestar en forma de insuficiencia ventricular derecha lentamente progresiva sin episodios de taquicardia ventricular (3). En el diagnóstico de ésta enfermedad hoy día contamos con técnicas de imagen no invasivas (ecocardiograma (4) y resonancia magnética nuclear) (5) que aportan información suficiente en la mayor parte de los casos, y hacen innecesaria la realización de biopsia endomiocárdica y ventriculografía derecha. Se ha publicado un informe6 que define los criterios para el diagnóstico de ésta enfermedad (Tabla I) basándose en la identificación de anomalías estructurales, la sustitución del tejido miocárdico por tejido fibroadiposo, alteraciones en el electrocardiograma, la existencia de taquicardia ventricular con morfología de bloqueo de rama izquierda e historia familiar. El tratamiento de ésta enfermedad consiste en suprimir las arritmias ventriculares, evitar la muerte súbita, y en algunos casos raros, tratar la insuficiencia cardiaca derecha. En un informe de 1995 la OMS/International Society and Federa tion of Cardiology Task Force, reevaluó la clasificación etiológica de las miocardiopatías, y consideró que la MAVD constituye una categoría aparte. Creemos que es de interés divulgar este caso con el fin de recordar que este tipo de miocardiopatía debe ser sospechada sistemáticamente en pacientes con arritmias ventriculares que tengan un origen en el ventrículo derecho, pero también en aquellos con una clínica de insuficiencia cardiaca derecha lentamente progresiva sin episodios de arritmias (7).

\section{Bibliografía}

1. Lobo FV, Heggtveit A, Butany J, Silver MD, Edwards JE. Right ventricular dysplasia: morphological findings in 13 cases. Can Cardiol 1992; 8: 261-268.

2. Corrado D, Basso C, Thiene G, et al. Spectrum of Clinicopathologic Manifestations of Arrhythmogenic Right Ventricular Cardiomyopathy/Dysplasia: A Multicenter Study. J Am Coll Cardiol 1997; 30: 1512-20.

3. Higuchi S, Caglal NM, Shimada R, Yamada A, Takeshita A, Nakamura M. 16 years follow-up of arrhythmogenic right ventricular disease. Am Heart J 1984; 106: 1365-1365.

4. Baran A, Nanda N, Falkoff M, Barold M, Gallagher JJ. Two dimensional echocardiography detection of arrhythmogenic right ventricular dyspla- sia. Am Heart J 1982; 103: 1066-1067.

5. Ricci C, Longo R, Pagnan L, Dalla Palma L, Pinamonti B, Camerini F et al. Magnetic resonance imaging in right ventricular dysplasia. Am J Cardiol 1992; 70: 1589-1595.

6. Richardson P, Mckenna WJ, Bristow M, et al. Report of the 1995 World Health Organization/International Society and Federation of Cardiology Task Force on the definition and classification of cardiomyopathies. Circulation 1996; 93: 841-2.

7. Brugada J, Mont L, Brugada R. Displasia arritmogénica del ventrículo derecho. Rev Esp Cardiol 1997; 50: 541-547. 